\title{
Semiparametric Diffusion Estimation and Application to a Stock Market Index
}

\author{
Wolfgang Härdle ${ }^{1}$, Torsten Kleinow ${ }^{1}$, Alexander Korostelev ${ }^{2}$, \\ Camille Logeay ${ }^{3}$, Eckhard Platen ${ }^{4}$ \\ ${ }^{1}$ Humboldt-Universität zu Berlin, Institut für Statistik und Ökonometrie, Spandauer \\ Strasse 1, 10178 Berlin, Germany, E-mail: kleinow@wiwi.hu-berlin.de \\ ${ }^{2}$ Wayne State University, Detroit, USA \\ 3 Deutsches Institut für Wirtschaftsforschung, Berlin, Germany \\ ${ }^{4}$ University of Technology Sydney, School of Finance and Economics, PO Box 123 \\ Broadway, Sydney, NSW 2007, Australia
}

This version: April 26, 2001

\begin{abstract}
The analysis of diffusion processes in financial models is crucially dependent on the form of the drift and diffusion coefficient functions. A methodology is proposed for estimating and testing coefficient functions for ergodic diffusions that are not directly observable. It is based on semiparametric and nonparametric estimates. The testing is performed via the wild bootstrap resampling technique. The method is illustrated on S\&P 500 index data.
\end{abstract}

JEL classification: C51, C52, G22

Keywords: Diffusion, Identification, Continuous-time financial models, Semiparametric methods, Kernel smoothing, Bootstrap

\section{Introduction}

The analysis of time series and diffusion models with stochastic variance and covariance has been very intense in the last decade. In particular, the research on stochastic volatility models in finance has been driven in several directions, see Ghysels, Harvey \& Renault (1996) and Frey (1997) for a survey. One direction is related to modern continuous time finance, see Genon-Catalot, Jeantheau \& Larédo (2000), where one has realized the necessity to relate empirically observed volatility or implied volatility data to theoretically derived stochastic volatility models. Another direction focuses on time series models for asset price and volatility which provide a well established framework for testing and modeling nonlinear variance structures in finance, see Engle \& Bollerslev (1986).

As shown by Nelson (1990) and Duan (1997), standard time series models, such as ARCH, GARCH etc., converge for vanishing time step size between observations towards corresponding diffusion models. These diffusion models are fully characterized by their drift and diffusion coefficient functions. The particular choice of the time discretization is not essential for a discrete time approximation of a diffusion process as long as the maximum time step size is small enough. Since real data are very frequently observed it is natural to 
interpret these as values of a discretely observed diffusion process or those of a corresponding discrete time approximation of a diffusion. The main statistical task in such a diffusion approach is the identification of the underlying drift and diffusion coefficient functions.

To handle this task in standard situations, a well developed statistical theory is now available. Parameter estimation methods for discretely observed stationary diffusion processes have been derived, for instance, by Bibby \& Sørensen (1995), Hansen \& Scheinkman (1995), Ait-Sahalia (1996), Gallant \& Tauchen (1996) and Kessler \& Soerensen (1999). Hansen, Scheinkman \& Touzi (1998) consider a nonparametric method based on the spectral decomposition of the conditional expectation operator to identify the drift and diffusion coefficients. Jiang \& Knight (1997) propose nonparametric estimators for the drift and diffusion coefficient, that are based on the approximation of the local time process and the estimation of the marginal density of the diffusion. A crucial assumptions for most of the developed estimation techniques is that a stationary diffusion is directly observed. Unfortunately, in reality this assumption is often not fulfilled. For instance, Genon-Catalot et al. (2000) consider a stochastic volatility model where the observed stock price depends on a hidden volatility process, which is itself a stationary diffusion.

We study here a case where a non-stationary diffusion process, an index, is observed. To be able to apply methods that rely on ergodicity we express the observed process as the product of an ergodic process and a smooth function of time. This smooth function is interpreted as average growth of the index. Due to the unknown impact of the average growth on the observed data, the ergodic part of our model is not directly observable. The proposed methodology combines recently developed nonparametric and parametric methods in order to estimate and probe the drift and diffusion coefficients of the ergodic process.

To illustrate our methodology we concentrate here on the empirical analysis of a particular stock market index, the S\&P 500. The statistical analysis of stock prices, exchange rates etc. is similar but not in the focus of this paper. We concentrate here on the case where an index is modeled by a scalar diffusion process.

The framework of Platen (2000) fully characterizes a financial market by the specification of the different denominations of the, so called, best benchmark portfolio. The stock index and the index benchmarked stock prices can be interpreted as denominations of the best benchmark portfolio. As a consequence, exchange prices are ratios of corresponding denominations of the best benchmark portfolio. Furthermore, this portfolio represents the optimal growth portfolio, see Karatzas \& Shreve (1998). A well diversified market index, as the S\&P 500, comes close to the optimal growth portfolio. For this reason, the inference for the index is also the first step in the statistical analysis of an exchange rate or stock price.

We assume that an appropriately normalized index process $X=\{X(t), t \geq$ $0\}$ can be interpreted as an ergodic process. Based on this assumption we focus on the inference of this normalized process $X$ instead of the index $S=\{S(t), t \geq$ $0\}$ itself. This allows us to direct our attention towards the identification of the drift and diffusion coefficient functions of an ergodic diffusion. In Figure 1 we plot the S\&P 500 index $S$ with daily data from 1977 to 1997 together with an average index $\bar{S}=\{\bar{S}(t), t \geq 0\}$. Such an average index $\bar{S}$ can be obtained in different ways. For instance, it could be exogenously given by a 
function of economic and financial quantities, i.e. inflation rate, growth rate of the domestic product, interest rate, etc.. It could also be derived by a kernel smoothing procedure, with an appropriate bandwidth or filter length $h$. This is the choice which we will study in this paper.

We construct the normalized index $X$ by dividing the original index $S$ by the above described average index $\bar{S}$, that is

$$
X(t)=\frac{S(t)}{\bar{S}(t)}
$$

for $t \geq 0$. The resulting normalized index $X$, derived via a kernel smoother, is shown in Figure 1. Its path resembles that of a stationary diffusion process. Note in the middle of our plot the sudden decline caused by the $1987 \mathrm{crash}$, which we do not remove from our sample.

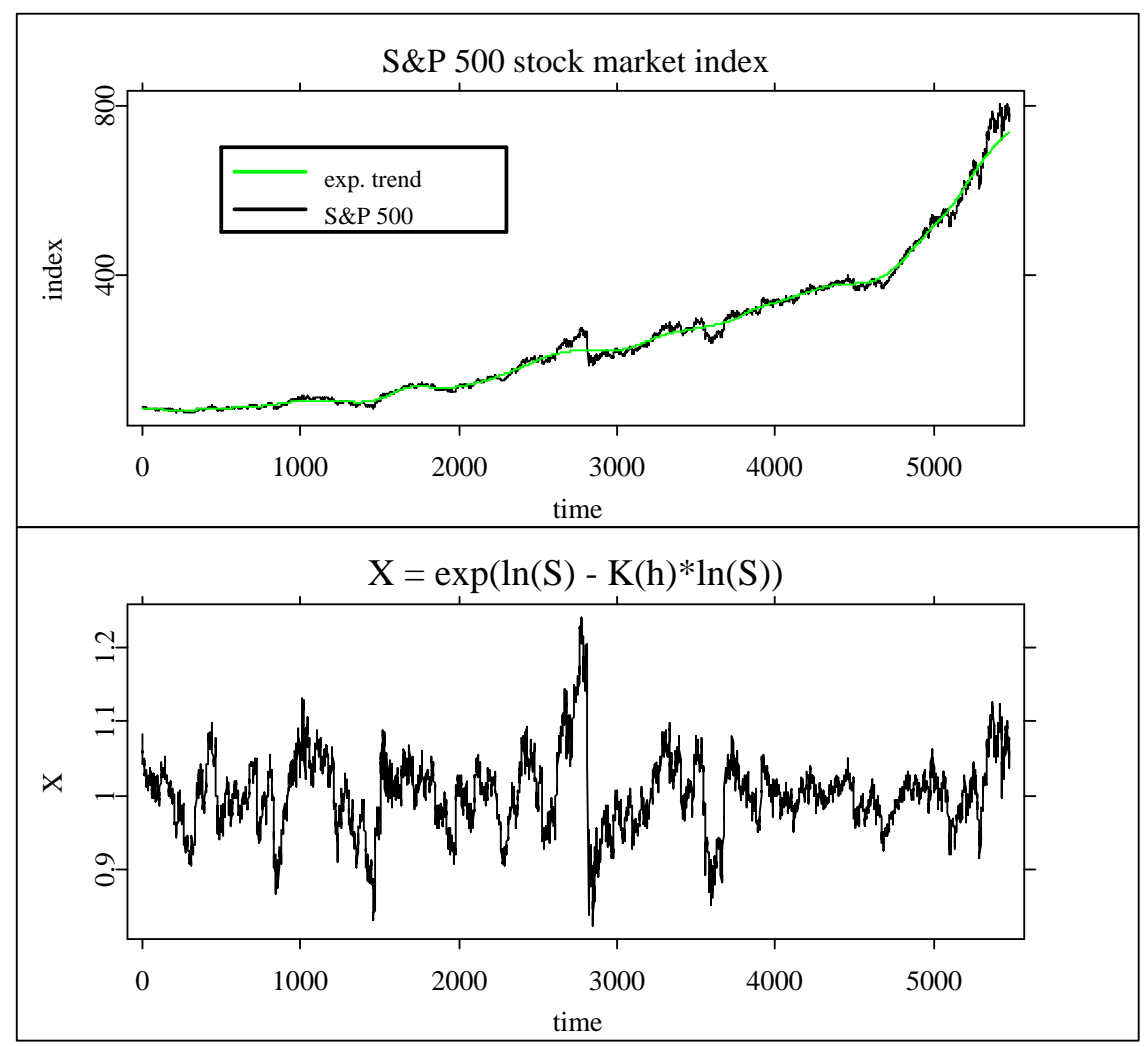

Figure 1: S\&P 500 index $S$, average index $\bar{S}$ and normalized index $X$

We assume for the value of the index $S(t)$ at time $t$ a representation of the form

$$
S(t)=S(0) Z(t) \exp \left\{\int_{0}^{t} \eta(s) d s\right\}
$$


for $t \geq 0$. Here $\eta(t)$ is interpreted as the deterministic, time dependent growth rate of the index at time $t$. Furthermore $Z(t)$ denotes the value of a positive ergodic diffusion process $Z$ at time $t$, that means, $Z$ solves the Itô stochastic differential equation (SDE)

$$
d Z(t)=m\{Z(t)\} d t+\sigma\{Z(t)\} d W(t)
$$

for $t \geq 0$. Here $W=\{W(t), t \geq 0\}$ denotes a standard Wiener process and $m\{$. and $\sigma\{$.$\} are the drift and diffusion coefficient functions. Due to the factor S(0)$ we assume that $Z$ is stable about 1 , which models a mean reverting behavior. On the other hand, $Z(t)$ has to be positive for all $t \geq 0$.

To make our parametric model specific we may choose for $Z$ a square root process, that is positive and stable about an equilibrium reference level. The square root process is also known as the Cox-Ingersoll-Ross (CIR) process, see Cox, Ingersoll \& Ross (1985). The functional form (1.2) that models the index is a special case of the minimal market model (MMM) proposed in Platen (2000).

Another parametric model arises if we choose

$$
Z(t)=\exp \{U(t)\}
$$

with an Ornstein-Uhlenbeck process $U=\{U(t), t \geq 0\}$. This leads us to the exponential of an Ornstein-Uhlenbeck process as index model. Such a model has been used, for instance, in Föllmer \& Schweizer (1993), Platen \& Rebolledo (1996) and Fleming \& Sheu (1999).

To compute the average index $\bar{S}$ in (1.1), we apply a kernel smoother to the logarithm of $S$ and then calculate $\bar{S}$ as the exponential of this smoothed process. This removes the average deterministic growth in (1.2). For the analysis of the resulting normalized index we have to take into account that the residuals $\ln S-\ln \bar{S}$ are corrupted by the smoother. This will be shown in detail later on. It means, that the normalized index $X$ is not a diffusion and in particular it does not equal the diffusion $Z$. For this reason we cannot directly apply estimation methods for discretely observed diffusions. From the statistical point of view we are faced with a nonparametric regression model with error terms that are not independent and identically distributed but are the discrete observations of a diffusion process. The analysis of these error terms and the clarification of their relationship to $Z$ is a main task in this paper.

We remark, that the index process $S$ is itself a diffusion. When $Z$ is specified according to (1.4) with an Ornstein-Uhlenbeck process $U$, Itô's formula yields the representation

$$
d S(t)=\{\eta(t)-\beta\} S(t) d t+\gamma \exp \left\{\int_{0}^{t} \eta(s) d s\right\} d W(t)
$$

for $t \geq 0$. The parameters $\beta, \gamma$ and $\eta$ cannot be easily separated in this representation. For this reason, we develop a statistical methodology for models that are based on the representation (1.2).

In Section 2.1 we introduce the parametric model for $Z$. The kernel smoothing and the computation of $X$ is described in Section 2.2. The choice of the kernel and bandwidth and its influence on the average index is discussed in Section 2.3 together with the corresponding parameter estimation methods. Section 2.4 introduces discrete time approximations of diffusion processes and Section 
2.5 describes the nonparametric estimation of drift and diffusion coefficient functions. In Section 2.6, a parametric model is tested versus a purely nonparametric alternative. This test is carried out by the bootstrap technique described in Appendix A.3. In Section 3 and 4 we apply the introduced methodology to S\&P 500 data and also in a simulation study.

We remark that the proposed methodology applies directly to situations, where normalized data can be modeled by an ergodic diffusion process. Emphasis is here given to the case of an Ornstein-Uhlenbeck process, and results on the influence of the kernel smoother are included for this case.

\section{Statistical Methodology for a Normalized Dif- fusion}

\subsection{Parametric Models}

As discussed in the introduction, one can, in principle, use various parametric ergodic diffusion models. Let us mention two examples. Both of them have mean reverting drift coefficients. In the case where the squared diffusion coefficient has the form

$$
\sigma^{2}(z)=\nu^{2} z, \quad z>0,
$$

with a positive constant $\nu$, we obtain in (1.2) a square root process $Z$. Here we assume that $Z$ satisfies the SDE

$$
d Z(t)=\{\psi-\varphi Z(t)\} d t+\nu \sqrt{Z(t)} d W(t)
$$

for $t \geq 0$ and with $\psi>\nu^{2} / 2, \varphi>0$. Note that a stationary and ergodic solution of (2.2) exists with the expected value $\mu_{\infty}=E[Z]=\psi / \varphi>0$. Since the ratio $Z(t) / \mu_{\infty}$ is again a square root process and any constant term can be absorbed by $S(0)$ in $(1.2)$, one can for simplicity assume that $\mu_{\infty}=E[Z]=1$. This choice leads us to the SDE

$$
d Z(t)=\varphi\{1-Z(t)\} d t+\nu \sqrt{Z(t)} d W(t) .
$$

for $t \geq 0$.

We obtain a second example for an ergodic diffusion by defining $Z$ as in (1.4), where $U$ denotes the well-known Ornstein-Uhlenbeck process with

$$
d U(t)=-\beta U(t) d t+\gamma d W(t) .
$$

for $t \geq 0$. Since $U$ fluctuates about its reference level 0 and is ergodic, $Z$ as given in (1.4) is an ergodic, positive diffusion process fluctuating about 1.

\subsection{Kernel Smoothing}

Denote by $K_{h}$ a smoother with a kernel $K$ and a bandwidth $h$. The smoothing of any process is denoted by a convolution operator $(*)$. As mentioned before, the normalized index $X(t)$ in (1.1) can be defined by the exponential of the difference of

$$
L(t)=\ln \{Z(t)\}
$$


and its smoother $\left(K_{h} * L\right)(t)$, that is:

$$
X(t)=\exp \left\{\ln S(t)-\left(K_{h} * \ln S\right)(t)\right\}=\exp \left\{L(t)-\left(K_{h} * L\right)(t)\right\} .
$$

Equation (2.6) holds if we neglect the difference between the accumulated deterministic growth rate $\int_{0}^{t} \eta(s) d s$ in (1.2) and its smoother, this means:

$$
\int_{0}^{t} \eta(s) d s-\left(K_{h} * \int_{0}^{\cdot} \eta(s) d s\right)(t) \approx 0 .
$$

Here we arrive at a delicate point of our study. If we want to remove efficiently the deterministic growth rate in (1.2), then the value $h$ should be chosen relatively small. Indeed, smaller values for $h$ reduce the bias. On the other hand, the smaller the value of $h$ is chosen, the more $X$ is corrupted by $K_{h} * L$ in (2.6).

The smoother $K_{h} * L$ is differentiable for differentiable kernels $K$ and thus of bounded variation. Due to the smoothing procedure $K_{h} * L$ involves also future information about $L$. Thus $X$ is not a diffusion process. For this reason, we cannot treat $\ln X(t)$ in (2.6) as the logarithm of a square root process or as an Ornstein-Uhlenbeck process. A more detailed analysis of $X$ has therefore to be performed. This is the objective of the next section. However note, in the case when $\bar{S}$ is obtained exogenously and not by a smoothing procedure, $X$ might still be a diffusion.

\subsection{Estimation of Parameters}

In this section we assume that the only observations available are those of

$$
\ln X(t)=L(t)-\left(K_{h} * L\right)(t)
$$

in (2.6) and that $L$ is the Ornstein-Uhlenbeck process $U$ given in (2.4). The estimation problem that we now consider is that for the parameters $\beta$ and $\gamma$ in (2.4). In principle the value of $\gamma$ can be restored from the quadratic variation of either $Z(t)$ or $L(t)$. For differentiable kernels $K_{h}$ in (2.6), the process $\left(K_{h} * L\right)(t)$ is also differentiable. For this reason it holds that

$$
\lim _{\Delta t \rightarrow 0} \sum_{i=1}^{n}\{\ln X(i \Delta t)-\ln X(i \Delta t-\Delta t)\}^{2} \stackrel{L^{2}}{=} \int_{0}^{T} d<L>_{t}
$$

for $n=T / \Delta t$. Here $d\langle L\rangle_{t}$ denotes the differential of the quadratic variation of the process $L$ at time $t$. Empirical results confirm that the quadratic variation is not sensitive to the choice of $h$. For more details on that see Table 1 . The following formula provides a stable estimate of $\gamma^{2}$ in the form

$$
\begin{aligned}
\hat{\gamma}^{2} & =T^{-1} \sum_{i=1}^{n}\{\ln X(i \Delta t)-\ln X(i \Delta t-\Delta t)\}^{2} \\
& \approx T^{-1} \int_{0}^{T} d<L>_{t} .
\end{aligned}
$$

To estimate the speed of adjustment parameter $\beta$ in (2.4) we could use the well-known form of the stationary variance of the Ornstein-Uhlenbeck process $L$. Along with (2.9) this would result in a first estimator of $\beta$ with

$$
\hat{\beta}_{1}=\hat{\gamma}^{2} /(2 \operatorname{Var}[L]) \text {. }
$$


Unfortunately, the substitution of $\operatorname{Var}[L]$ by $\operatorname{Var}[\ln X]$ makes $\hat{\beta}_{1}$ strongly dependent on $h$. Indeed, the variance

$$
\operatorname{Var}[\ln X]=\operatorname{Var}\left[L-K_{h} * L\right]
$$

increases as $h$ grows, and only for very large values of $h$ we can expect that $\operatorname{Var}[\ln X] \approx \operatorname{Var}[L]$.

It is not just the variance of the random process $\ln X$ that changes with $h$. Also its autocorrelation function depends on the bandwidth $h$. The correlation between the values of the process $\ln X$, distant by a constant time length $\tau>0$, diminishes as $h$ decreases. For this reason we propose a selection method for $h$ based on the simultaneous estimation of $\beta$ from the variance and from the autocorrelation function of the process $\ln X$. The idea is simple, if for each value of $h$ there are two different estimates of the same parameter $\beta$, then the best choice of $h$ is considered to be that, which brings these estimates as close as possible to each other.

The autocorrelation function $\rho^{(L)}(\tau)$ of the Ornstein-Uhlenbeck process $L$ equals

$$
\rho^{(L)}(\tau)=e^{-\beta \tau}
$$

for $\tau>0$. Thus, $\beta$ represents the absolute value of the slope of this function at zero. Hence another estimate of $\beta$ from the observations of $L$ would be

$$
\hat{\beta}_{2}=\left.\left|\frac{\partial^{+}}{\partial \tau} \rho^{(L)}(\tau)\right|\right|_{\tau=0},
$$

where

$$
\frac{\partial^{+}}{\partial \tau} \rho(\tau)=\lim _{s \rightarrow 0, s>0} \frac{\rho(\tau+s)-\rho(\tau)}{s}
$$

for $\tau \geq 0$ denotes the right hand derivative of $\rho$ with respect to $\tau$.

Unfortunately, the estimator in (2.13) is not feasible since $L$ is not observed. In Appendix A.1 we show for the process $\ln X$ that its stationary variance is asymptotically

$$
\operatorname{Var}[\ln X]=\frac{\gamma^{2}}{2 \beta}\left(1-\frac{c_{K}}{\beta h}+O\left(h^{-2}\right)\right) \text { as } h \rightarrow \infty,
$$

where the constant $c_{K}$ depends on the kernel $K$. Furthermore, we prove in Appendix A.1 for the autocorrelation function $\rho_{h}^{(\ln X)}(\tau)$ of $\ln X$ the asymptotics

$$
\begin{aligned}
\rho_{h}^{(\ln X)}(\tau) & =\operatorname{Corr}[\ln X(\tau) ; \ln X(0)] \\
& =\frac{e^{-\beta \tau}-\frac{c_{K}}{\beta h}+O\left(\tau h^{-2}\right)}{1-\frac{c_{K}}{\beta h}+O\left(h^{-2}\right)}, \quad \tau \geq 0
\end{aligned}
$$

as $h \rightarrow \infty$ with the same constant $c_{K}$ as in (2.14). In Appendix A.1 this constant is calculated for the rectangle and the Epanechnikov kernels.

It follows from equation (2.14) that the first-order approximation of the stationary variance of $\ln X$ is

$$
\operatorname{Var}[\ln X] \approx\left\{1-\frac{c_{K}}{\beta h}\right\} \operatorname{Var}[L]
$$


By (2.15), the slope of the autocorrelation of $\ln X$ at zero is asymptotically

$$
\left.\left|\frac{\partial^{+}}{\partial \tau} \rho_{h}^{(\ln X)}(\tau)\right|\right|_{\tau=0} \approx \frac{\beta}{1-\frac{c_{K}}{\beta h}}
$$

as $h \rightarrow \infty$. Thus this slope is steeper than that of $\rho^{L}$ at $\tau=0$.

The immediate consequence of (2.14) and (2.15) is that the formulas (2.10) and (2.13) for $\hat{\beta}_{1}$ and $\hat{\beta}_{2}$, respectively, have to be modified if the process $\ln X$ rather than $L$ is observed. In Appendix A.1 we show that the correct modification is provided by the expressions

$$
\hat{\beta}_{1}(h)=\frac{\hat{\gamma}^{2}}{2 \operatorname{Var}[\ln X]}-\frac{c_{K}}{h}
$$

and

$$
\hat{\beta}_{2}(h)=\left.\left|\frac{\partial^{+}}{\partial \tau} \rho_{h}^{(\ln X)}(\tau)\right|\right|_{\tau=0}-\frac{c_{K}}{h},
$$

respectively. Finally, our method for the selection of $h$ is based on the following balance equation

$$
\hat{\beta}_{1}(h)=\hat{\beta}_{2}(h)
$$

which equals both estimates.

After $h$ is chosen, we need to restore the process $L$, which is needed in the remaining nonparametric and parametric analysis. From (2.6), proceeding formally, one arrives at the following iterative formula:

$$
\begin{aligned}
L=\ln X+K_{h} * L & =\ln X+K_{h} *\left(\ln X+K_{h} * L\right) \\
& =\ldots \\
& =\ln X+K_{h} * \ln X+K_{h} * K_{h} * \ln X+\ldots
\end{aligned}
$$

The justification for the restoration formula (2.21) comes from the fact that if one neglects the boundary effects, the smoothing operator $K_{h}$ is a contracting operator in $L_{2}$, as shown in Appendix A.1. In the practical application of (2.21), we rely on the fact that the smoother of the original process $L$ is close to the smoother of $L-K_{h} * L$. In practice, only one or two convolutions are meaningful. After the restoration process is completed, the parameter $\beta$ can be estimated directly from $L$ by (2.10).

We were able to establish in this paper the above correction terms for the Ornstein-Uhlenbeck process. One could, in principle, estimate parameters also under the assumption that $X$ itself is a square root process or another ergodic diffusion. However, if the average index $\bar{S}$ is calculated via a smoothing procedure, a similar bandwidth selection method has to be developed. At that stage this is left for future research.

\subsection{Discrete Time Approximation of a Diffusion}

We interpret the process $Z(t)$, which appears in (1.2), as a positive ergodic scalar diffusion process that is the solution of the $\operatorname{SDE}(1.3)$. The drift $m$ : 
$[0, \infty) \mapsto(-\infty, \infty)$ and the diffusion coefficient $\sigma:[0, \infty) \mapsto[0, \infty)$ in (1.3) are assumed to be sufficiently regular, such that a unique solution of (1.3) exists.

For the existence of an ergodic solution of (1.3) the drift and the diffusion coefficient must satisfy some ergodicity conditions, see for instance Bibby \& Sørensen (1995). The most important condition is that the stationary Kolmogorov forward equation

$$
\frac{1}{2} \frac{\partial}{\partial z}\left\{\sigma^{2}(z) p_{0}(z)\right\}-m(z) p_{0}(z)=0
$$

must have a solution $p_{0}(z)$ which is then up to a constant the stationary probability density.

In particular, the above conditions hold for the exponential of an OrnsteinUhlenbeck process with a log normal stationary distribution and for the square root process which has a Gamma-distribution as stationary distribution.

Let us assume that the diffusion process $Z$ is observed at discrete times $t_{i}=i \Delta, i=1,2, \ldots$, with a time step size $\Delta>0$. Here we suppose that $\Delta$ is small or, more precisely, will tend to zero asymptotically. Under rather weak assumptions, see Kloeden \& Platen (1999), on the functions $m$ and $\sigma^{2}$, it can be shown that the Euler approximation

$$
Z^{\Delta}(t)=Z^{\Delta}(0)+\int_{0}^{t} m\left\{Z^{\Delta}\left(t_{i_{s}}\right)\right\} d s+\int_{0}^{t} \sigma\left\{Z^{\Delta}\left(t_{i_{s}}\right)\right\} d W(s)
$$

with $t_{i_{s}}=\max \left\{t_{i}, t_{i} \leq s\right\}$, converges in a mean square sense to $Z$ as $\Delta \rightarrow 0$, i.e.,

$$
\lim _{\Delta \rightarrow 0} E\left[\sup _{0 \leq t \leq T}\left|Z^{\Delta}(t)-Z(t)\right|^{2}\right]=0, \quad T>0 .
$$

From now on, we assume that a discrete time approximation $Z^{\Delta}$ exists in the form of (2.22), and that the convergence property (2.23) holds. For the purposes of this paper, $\Delta$ will always be considered to be small enough so that one can substitute $Z$ by $Z^{\Delta}$ without any major error in our interpretation of the observed data. The concrete choice of $\Delta$ does not matter since all the relevant information about the model is contained in the drift $m$ and diffusion coefficient $\sigma$. As the estimates of moments of higher order terms in the case of the daily observed $\mathrm{S} \& \mathrm{P} 500$ show, the step size $\Delta$ corresponding to the given daily observations is small enough so that the difference between $Z^{\Delta}\left(t_{i}\right)$ and $Z\left(t_{i}\right)$ is indeed negligible. Thus we interpret the increments of the observed data as those of the Euler approximation (2.22), that is

$$
Z^{\Delta}\left(t_{i+1}\right)-Z^{\Delta}\left(t_{i}\right)=m\left\{Z^{\Delta}\left(t_{i}\right)\right\} \Delta+\sigma\left\{Z^{\Delta}\left(t_{i}\right)\right\}\left\{W\left(t_{i+1}\right)-W\left(t_{i}\right)\right\}
$$

for $i=0,1, \ldots$ Note that the observations $\left(Z^{\Delta}\left(t_{i}\right)\right), i=0,1, \ldots$ form a state dependent time series.

\subsection{Nonparametric Estimation}

For two consecutive observations $Z^{\Delta}\left(t_{i}\right)$ and $Z^{\Delta}\left(t_{i+1}\right)$ of the above time series we define the increment

$$
Y_{i}=Z^{\Delta}\left(t_{i+1}\right)-Z^{\Delta}\left(t_{i}\right)=m\left\{Z^{\Delta}\left(t_{i}\right)\right\} \Delta+\sigma\left\{Z^{\Delta}\left(t_{i}\right)\right\} \sqrt{\Delta} \varepsilon_{i}
$$


with independent standard Gaussian random variables

$$
\varepsilon_{i}=\frac{W\left(t_{i+1}\right)-W\left(t_{i}\right)}{\sqrt{\Delta}} \sim \mathcal{N}(0,1) .
$$

It is now possible to use the increments $Y_{i}$ to estimate the functions $m\{$.$\} and$ $\sigma^{2}\{$.$\} . In particular, we apply the local linear nonparametric method explained$ in Fan \& Gijbels (1996) and Härdle \& Tsybakov (1997). To justify the use of this method, the time series $Z^{\Delta}\left(t_{i}\right)$ has to be ergodic and to meet some technical conditions, see Härdle \& Tsybakov (1997). The square root process and the Ornstein-Uhlenbeck process are examples, where the discrete time approximation (2.25) satisfies these conditions.

In this nonparametric framework we obtain the drift function estimator

$$
\hat{m}_{h_{1}}(z)=\frac{1}{\Delta} \hat{\beta}_{0}(z)
$$

with

$$
\begin{aligned}
\hat{\beta}(z) & =\left(\begin{array}{c}
\hat{\beta}_{0}(z) \\
\hat{\beta}_{1}(z)
\end{array}\right) \\
& =\operatorname{argmin}_{b_{0}, b_{1}}\left(\sum_{i=1}^{n}\left\{Y_{i}-b_{0}-b_{1}\left(Z^{\Delta}\left(t_{i}\right)-z\right)\right\}^{2} K_{h_{1}}\left(z-Z^{\Delta}\left(t_{i}\right)\right)\right) .
\end{aligned}
$$

The bandwidth $h_{1}>0$ is chosen with respect to the Silvermans rule of thumb, see Härdle (1990). The Gaussian density function is used as the kernel.

We apply for the squared diffusion function $\sigma^{2}(z)$ a two-step estimation. First we compute from the above drift function estimator (2.26) the values $\hat{m}_{h_{1}}\left\{Z^{\Delta}\left(t_{i}\right)\right\}$. In the second step we use the squared diffusion function estimator

$$
\hat{\sigma}_{h_{1}}^{2}(z)=\frac{1}{\Delta} \hat{\delta}_{0}(z)
$$

with

$$
\begin{aligned}
\hat{\delta}(z)= & \left(\begin{array}{c}
\hat{\delta}_{0}(z) \\
\hat{\delta}_{1}(z)
\end{array}\right) \\
= & \operatorname{argmin}\left(\sum _ { i = 1 } ^ { n } \left\{\left(Y_{i}-\Delta \hat{m}_{h_{1}}\left\{Z^{\Delta}\left(t_{i}\right)\right\}\right)^{2}\right.\right. \\
& \left.\left.-\delta_{0}-\delta_{1}\left(Z^{\Delta}\left(t_{i}\right)-z\right)\right\}^{2} K_{h_{1}}\left(z-Z^{\Delta}\left(t_{i}\right)\right)\right)
\end{aligned}
$$

and bandwidth $h_{1}>0$. For details and the asymptotic properties of these estimators and the construction of corresponding confidence bands we refer to Appendix A.2 and A.3.

The application of the above methodology to the data $Z^{\Delta}$ yields corresponding nonparametric estimates with an estimated squared diffusion coefficient function as well as a drift coefficient function along with corresponding confidence bands as we demonstrate below. 


\subsection{Testing the Parametric Model}

We construct tests to compare the nonparametric estimates of Section 2.5 for $m\{$.$\} and \sigma^{2}\{$.$\} to parametric forms, for example the coefficients of an Ornstein-$ Uhlenbeck process.

To derive the null hypotheses in the case when $Z$ is the exponential of an Ornstein Uhlenbeck process, we apply Itô's formula to $Z(t)=\exp \{U(t)\}$. Here $U$ satisfies (2.4) and one obtains

$$
\begin{aligned}
d Z(t) & =d(\exp \{U(t)\}) \\
& =Z(t)\left\{-\beta \ln Z(t)+\frac{1}{2} \gamma^{2}\right\} d t+\gamma Z(t) d W(t)
\end{aligned}
$$

for $t \geq 0$. The null hypotheses of the tests are therefore

$$
H_{0}(m): m(z)=z\left\{-\beta \ln z+\frac{1}{2} \gamma^{2}\right\}
$$

and

$$
H_{0}\left(\sigma^{2}\right): \sigma^{2}(z)=\gamma^{2} z^{2}
$$

while the alternative is nonparametric.

We construct confidence bands with the bootstrap method. The idea is to bootstrap the original discrete time series and estimate each time the drift and squared diffusion coefficients nonparametrically as described in Section 2.5. With these estimates one can then construct confidence bands for the two functions.

We choose the bootstrap method because it leads to better coverage probabilities than, for instance, a Gaussian approximation. In Neumann \& Kreiss (1998) it was shown for a time series similar to (2.25) that the coverage probability is of order $O\left(n^{-q}\right)$ for some $q>0$, where $n$ is the number of observations. A Gaussian approximation, see Hall (1985), leads to a coverage probability of order $O(1 / \ln (n))$.

The bootstrap method is described in Appendix A.3. The asymptotic results for the $(1-\alpha)$ confidence bands $K B(m)$ and $\left(K B\left(\sigma^{2}\right)\right)$, that is

$$
P\{m(z) \in K B(m)\} \rightarrow 1-\alpha
$$

and

$$
P\left\{\sigma^{2}(z) \in K B\left(\sigma^{2}\right)\right\} \rightarrow 1-\alpha
$$

respectively, are proved in Franke, Kreiss, Mammen \& Neumann (1998).

\section{Empirical Analysis of the S\&P 500}

We apply the methods introduced in Section 2 to daily observations of the S\&P 500 index from 31.12.1976 to 31.12 .1997 (5479 observations). The data are obtained from Thomson Financial Datastream.

For the kernel smoothing of $S$ we choose the Epanechnikov kernel. The constant $c_{K}$ that appears in the correction terms in (2.18) and (2.19) are known for this particular kernel, see Appendix A.1. 
As already mentioned in Section 2.3 the estimates for the parameter $\gamma$ calculated from formula (2.9) are small relative to 1 and do not change significantly with $h$. Table 1 shows the estimated values for different values of $h$. The variance of the process $X$ is also shown in that table. The small variance and the fact that $X$ is stable about 1 justifies to concentrate on the case of a geometric Ornstein-Uhlenbeck process defined by (1.4) and (2.4).

\begin{tabular}{l|rrrrr}
$h$ & 200 & 250 & 300 & 350 & 400 \\
\hline $\operatorname{Var}(X)$ & 0.0018303 & 0.0023465 & 0.0029246 & 0.0035622 & 0.0042183 \\
$\hat{\gamma}$ & 0.0090593 & 0.0090703 & 0.0090849 & 0.0090991 & 0.0091103
\end{tabular}

Table 1: Estimated values for $\gamma$ and the estimated variance of $X$ for different bandwidths $h$.

The next step in our analysis is the choice of $h$. Due to the long range of observations we apply a flexible bandwidth to the data. This flexible bandwidth was calculated by splitting the data in overlapping subintervals of different lengths and calculating an optimal fixed bandwidth for every subinterval. The bandwidth is chosen to be optimal with respect to the balance equation (2.20). To get a continuous optimal bandwidth function $h_{o p t}(t)$ we interpolate the resulting values. The function $t \mapsto h_{\text {opt }}(t)$ is shown in Figure 2. The fi-

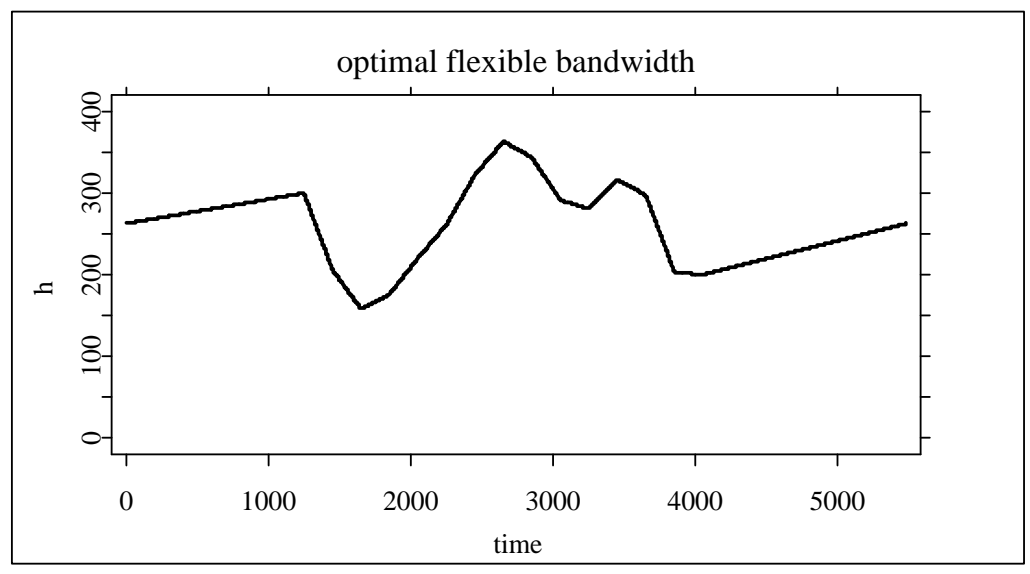

Figure 2: The optimal flexible bandwidth $h_{o p t}(t)$.

nal values for $\beta$ are $\hat{\beta}_{1}\left(h_{\text {opt }}\right)=0.010352$ and $\hat{\beta}_{2}\left(h_{\text {opt }}\right)=0.0089721$ and the ratio is $\hat{\beta}_{1}\left(h_{o p t}\right) / \hat{\beta}_{1}\left(h_{o p t}\right)=1.1538$. For fixed bandwidths in the range of $h_{\text {opt }}$ this ratio is given in Table 2. All these ratios are larger than those for $h_{\text {opt }}$ which justifies the use of the flexible bandwidth. The estimated value for $\gamma$ is $\hat{\gamma}\left(h_{\text {opt }}\right)=0.0091033$.

Now we are in the position to restore the path of the process $Z$ and to 


\begin{tabular}{l|rrrrr}
$h$ & 200 & 225 & 250 & 275 & 300 \\
\hline$\hat{\beta}_{1}(h)$ & 0.017913 & 0.01569 & 0.013826 & 0.012197 & 0.010776 \\
$\hat{\beta}_{2}(h)$ & 0.01328 & 0.012098 & 0.011092 & 0.0098626 & 0.0086047 \\
$\hat{\beta}_{1}(h) / \hat{\beta}_{2}(h)$ & 1.3489 & 1.2969 & 1.2465 & 1.2367 & 1.2523
\end{tabular}

Table 2: Estimated values for $\beta$ for different fixed bandwidths $h$.

estimate the parameters. We get the following estimates from the restored path

$$
\begin{aligned}
\hat{\beta}_{1} & =0.01003, & \hat{\beta}_{2} & =0.0093294, \\
\hat{\beta}_{1} / \hat{\beta}_{2} & =1.0751, & \hat{\gamma} & =0.0092454 .
\end{aligned}
$$

To finish the empirical analysis we apply the test procedure described in Section 2.6. Figure 3 shows the nonparametric estimates of the drift and squared diffusion coefficient of the restored process $Z$ together with the $90 \%$ confidence bands. The almost straight lines show the parametric estimates with respect to the estimated values of the restored process $Z$. The vertical lines enclose the interval where $99 \%$ of the observed data reside.

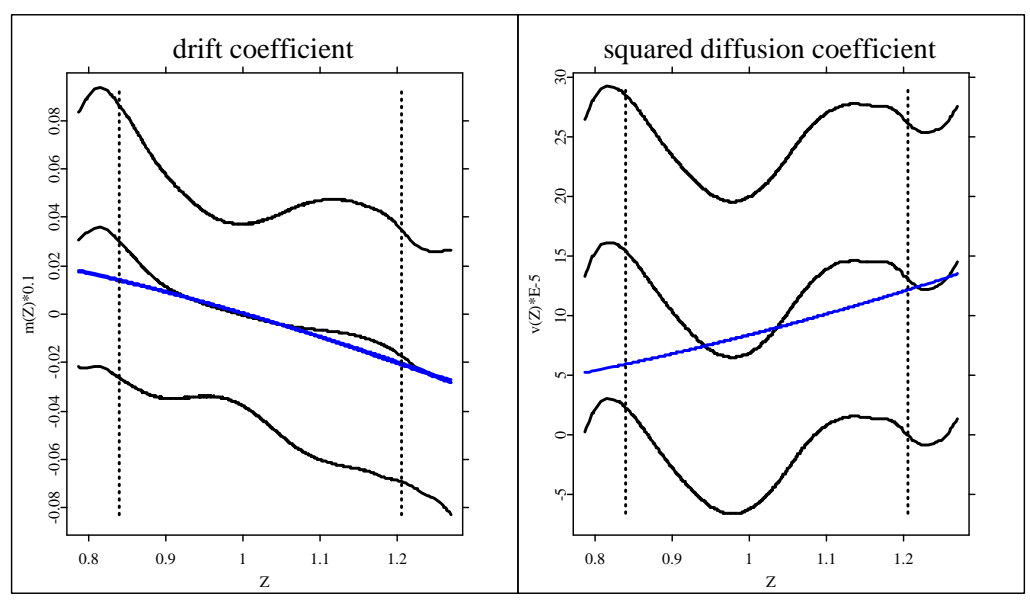

Figure 3: Nonparametric and parametric estimates of the drift $m($.$) and squared$ diffusion coefficient $\sigma^{2}($.$) with 90 \%$ confidence bands.

Both parametric functions are surely inside the confidence bands. Thus the null hypothesis of the geometric Ornstein-Uhlenbeck process cannot be rejected.

\section{Simulation Study}

We perform now a simulation study by applying the estimation methods introduced in Section 2.3 to simulated trajectories of the Ornstein-Uhlenbeck process $U$. The drift and diffusion parameters $\beta$ and $\gamma$ in (2.4) are estimated directly 
from the observations of $U$ as well as from the residual of a kernel smoothing procedure.

It is well known that the transition probability of an Ornstein-Uhlenbeck process is normal with conditional mean

$$
E\left[U_{t+\Delta} \mid U_{t}=u\right]=u e^{-\beta \Delta}
$$

and conditional variance

$$
\operatorname{Var}\left(U_{t+\Delta} \mid U_{t}=u\right)=\frac{\gamma^{2}}{-2 \beta}\left(e^{-2 \beta \Delta}-1\right) .
$$

Using this Gaussian transition probability we simulate 100 paths of the process $U$ with time step size $\Delta=1$. The true parameters are set to $\beta=0.01$ and $\gamma=0.01$, which correspond approximately to the empirical estimates for the S\&P 500 index in Section 3.

For the analysis of the directly observed process $U$ we apply three estimators for the speed of adjustment parameter $\beta$. Besides $\hat{\beta}_{1}$ and $\hat{\beta}_{2}$ introduced in (2.10) and (2.13), we use also the estimator

$$
\hat{\beta}_{3}=-\frac{1}{\Delta} \ln \frac{\sum_{i=1}^{n} U_{i-1} U_{i}}{\sum_{i=1}^{n} U_{i-1}^{2}},
$$

which is based on martingal estimating functions and was proposed in Bibby $\&$ Sørensen (1995). It is easy to see, that $\hat{\beta}_{3}$ is related to the autocorrelation function of $U$. For details about this estimator and the theory of martingal estimating functions we refer to Bibby \& Sørensen (1995) and the references therein. The diffusion coefficient is estimated via the slope of the quadratic variation, similarly as in (2.9).

The first row of Table 3 shows the means of the corresponding estimated values. In the second row the variance of the estimates are shown. We emphasize that the results are based on a directly observed simulated diffusion.

\begin{tabular}{rrrrrr}
$\hat{\beta}_{1}$ & $\hat{\beta}_{2}$ & $\hat{\beta}_{3}$ & $\gamma$ & $\hat{\beta}_{1} / \hat{\beta}_{2}$ & opt $h$ \\
\hline 0.01070 & 0.01018 & 0.01028 & 0.00996 & & \\
$4.589 \mathrm{e}-06$ & $3.695 \mathrm{e}-06$ & $4.541 \mathrm{e}-06$ & $1.059 \mathrm{e}-08$ & & \\
\hline 0.00964 & 0.00967 & & 0.00996 & 0.99435 & 295.0 \\
$7.161 \mathrm{e}-06$ & $6.258 \mathrm{e}-06$ & & $1.067 \mathrm{e}-08$ & 0.00248 &
\end{tabular}

Table 3: Estimated parameters.

Furthermore, we simulate the logarithm of the index in (1.2) $\ln S$ as the sum of a linear function and $U$. In a second step we calculate $\ln X$ as in (2.6) with the Epanechnikov kernel, see Appendix A.1. We then estimate from the simulated data the parameters $\beta$ and $\gamma$ of $U$ by the methods in (2.18), (2.19) and (2.9). This gives us an idea about the fixed sample behavior of these estimation methods when the residuals of a kernel smoothing procedure are observed instead of those of an Ornstein-Uhlenbeck process itself.

The estimated values calculated from the simulated trajectories of $\ln X$ are shown in the third and fourth row of Tables 3 . The results clearly demonstrate 
that the correction terms in (2.18) and (2.19) are necessary to obtain a reasonable estimated values. In the situation considered here, the correction terms equal each other and have approximately the value $c_{k} / h \approx 0.0061$, see Appendix A.1. Since the correction terms for $\hat{\beta}_{3}$ are not considered, we have not to report them in Table 3.

The table also shows the mean and the variance of the ratio $\hat{\beta}_{1} / \hat{\beta}_{2}$ used to select the bandwidth $\mathrm{h}$, see (2.20). The mean of the selected bandwidth $h$, which brings this ratio as close as possible to one, is given in the last column.

The second part of the simulation study treats the bootstrap procedure. We apply the bootstrap methodology as introduced in Appendix A.3 to a simulated path of an Ornstein-Uhlenbeck process $U$ following the dynamics in (2.4) with parameters $\beta=0.01, \gamma=0.01$ and $\Delta=1$. The values of the parameters are reasonable with respect to the empirical results for the $\mathrm{S} \& \mathrm{P} 500$. The number of observations is 5000 and the number of the bootstrapped series for the confidence bands is 160. The two plots in Figure 4 show the nonparametric estimators for the drift and squared diffusion coefficient together with their $90 \%$ confidence bands constructed by the bootstrap procedure. The plots also show the true parametric functions for the drift and diffusion coefficient. The dotted vertical lines are the empirical 0.005 and 0.995 quantiles of the stationary distribution of $\exp (U)$. If we only consider the range between these quantities, i.e. the range where $99 \%$ of the data reside, then both of the parametric functions remain inside the confidence bands. This means, the null hypotheses $H_{0}(m)$ and $H_{0}\left(\sigma^{2}\right)$ as in Section 2.6 cannot be rejected for data in this range.

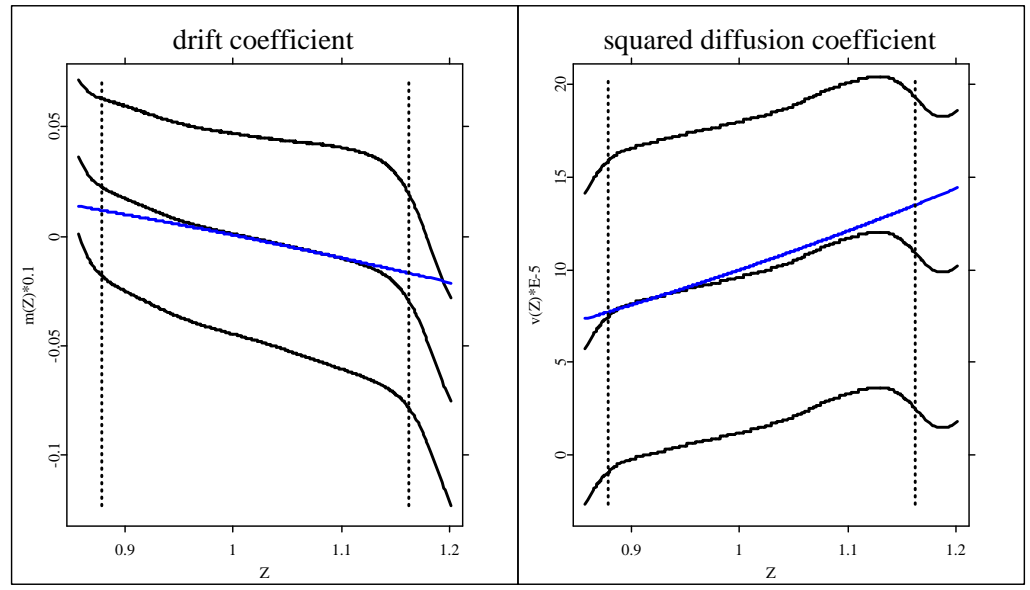

Figure 4: Nonparametric estimates for the drift and squared diffusion coefficients of a simulated geometric Ornstein-Uhlenbeck process, confidence bands and true functions. 


\section{Conclusion}

In this paper we modeled an index as the product of an ergodic diffusion and a deterministic growth process. In the first part we proposed a methodology that allows us to separate the estimation of the average growth of the index and that of the parameters of the ergodic diffusion. The general methodology was carried out for the particular case of an Ornstein-Uhlenbeck process. A challenge for future research is to establish similar estimation methods for parameters of a square root process and other ergodic diffusions. The derivation of the corresponding correction terms will be the key problem in such an approach.

In the second part of the paper we developed a semiparametric testing procedure for the drift and diffusion coefficient functions of an ergodic diffusion. The test is based on a comparison of the parametric forms of these functions to their nonparametric estimators. Finally, an empirical analysis of the S\&P 500 stock market index and a simulation study completed the paper.

\section{A Appendix}

\section{A.1 Parameter Estimation}

Let $U(t)$ be the Ornstein-Uhlenbeck process satisfying (2.4). Introduce the autocovariance of the process $U-K_{h} * U$ as

$$
\operatorname{Cov}(\tau)=\operatorname{COV}\left[\left(U-K_{h} * U\right)(\tau) ;\left(U-K_{h} * U\right)(0)\right], \quad 0<\tau<<h .
$$

Let

$$
\rho_{h}(\tau)=\frac{\operatorname{Cov}(\tau)}{\operatorname{Cov}(0)}
$$

be the autocorrelation function of $U-K_{h} * U$.

Proposition 1. (i) If $K(u)$ is the rectangle kernel, i.e., $K(u)=(1 / 2) \mathbb{I}(|u| \leq$ $1)$, then as $h \rightarrow \infty$ we have that

$$
\operatorname{Cov}(\tau)=\frac{\gamma^{2}}{2 \beta}\left(e^{-\beta \tau}-\frac{1}{\beta h}-\frac{1}{2 \beta h}(\tau / h)+O\left(h^{-2}\right)\right) .
$$

(ii) If $K(u)=(3 / 4)\left(1-u^{2}\right) \mathbb{I}(|u| \leq 1)$ is the Epanechnikov kernel, then

$$
\operatorname{Cov}(\tau)=\frac{\gamma^{2}}{2 \beta}\left(e^{-\beta \tau}-\frac{1.8}{\beta h}-\frac{1.5}{\beta h}(\tau / h)^{2}+O\left(h^{-2}\right)\right) .
$$

Proposition 2. Under the assumptions of Proposition 1, up to the terms of the magnitude $O\left(h^{-2}\right)$, the following equation holds for $\beta$ :

$$
\left.\frac{\partial^{+}}{\partial \tau} \rho_{h}(\tau)\right|_{\tau=0}=-\frac{\beta}{1-c_{K} /(\beta h)} .
$$

The solution of this equation is approximately

$$
\hat{\beta}_{2}=\left.\left|\frac{\partial^{+}}{\partial \tau} \rho_{h}(\tau)\right|\right|_{\tau=0}-\frac{c_{K}}{h}+O\left(h^{-2}\right)
$$


where $c_{K}=1$ if $K$ is the rectangle kernel, and $c_{K}=1.8$ if $K$ is the Epanechnikov kernel.

To the proof of Proposition 1. (i) Integrate the both sides of (2.4) with the rectangle kernel. The integration results in

$$
-\frac{1}{2 \beta h}\{U(t+h)-U(t-h)\}=\left(K_{h} * U\right)(t)-\frac{\gamma}{2 \beta h}\{W(t+h)-W(t-h)\},
$$

or

$$
\left(K_{h} * U\right)(t)=\frac{\gamma}{2 \beta h}(W(t+h)-W(t-h))-\frac{1}{2 \beta h}(U(t+h)-U(t-h)) .
$$

The autocovariance function equals

$$
\begin{aligned}
\operatorname{Cov}(\tau)= & E[U(\tau) U(0)]-E\left[U(\tau)\left(K_{h} * U\right)(0)\right] \\
& -E\left[U(0)\left(K_{h} * U\right)(\tau)\right]+E\left[\left(K_{h} * U\right)(\tau)\left(K_{h} * U\right)(0)\right] .
\end{aligned}
$$

The first term in the latter formula is $E[U(\tau) U(0)]=\left\{\gamma^{2} /(2 \beta)\right\} e^{-\beta \tau}$. With the help of (A.3) and the explicit representation

$$
U(t)=\int_{-\infty}^{t} \exp \{-\beta(t-s)\} \gamma d W(s)
$$

one finds by direct calculation that each of the negative terms on the right-hand side of (A.4) contributes

$$
-\frac{\gamma^{2}}{\left(2 \beta^{2} h\right)}+O\left(h^{-2}\right)
$$

while the covariance of $K_{h} * U$ adds up to

$$
\frac{\gamma^{2}(2 h-\tau)}{(2 \beta h)^{2}}+O\left(h^{-2}\right) .
$$

Combining these results, we arrive at (A.1).

(ii) Integrating (2.4), we find as in (A.3) that

$$
\left(K_{h} * U\right)(t)=-\frac{3}{2 \beta h^{3}} \int_{t-h}^{t+h}(s-t) U(s) d s+\frac{\gamma}{\beta}\left(K_{h} * W\right)(t) .
$$

It is straightforward to verify that the variance of the first term on the righthand side of (A.5) has the magnitude $O\left(h^{-2}\right)$ for $h$ large. This term is negligible as compared to the second one. As in part (i), we obtain

$$
\begin{aligned}
\operatorname{Cov}(\tau) & =\frac{\gamma^{2}}{2 \beta} e^{-\beta \tau}-2\left(\frac{3 \gamma^{2}}{4 \beta^{2} h}+O\left(h^{-2}\right)\right)+\frac{\gamma^{2}}{\beta^{2}} E\left[\left(K_{h} * W\right)(\tau)\left(K_{h} * W\right)(0)\right] \\
& =\frac{\gamma^{2}}{2 \beta} e^{-\beta \tau}-\frac{3 \gamma^{2}}{2 \beta^{2} h}+\frac{\gamma^{2}}{\beta^{2}}\left(\frac{3}{5}-\frac{3}{4}\left(\frac{\tau}{h}\right)^{2}\right)+O\left(h^{-2}\right)
\end{aligned}
$$

This proves (A.2).

To the proof of Proposition 2. The slope of the autocorrelation at zero follows from (A.1) and (A.2). Let

$$
A=\left.\left|\frac{\partial^{+}}{\partial \tau} \rho_{h}(\tau)\right|\right|_{\tau=0},
$$


then for $\hat{\beta}_{2}$ the quadratic equation $\hat{\beta}_{2}^{2} h-\hat{\beta}_{2} h A+c_{K} A=0$ holds with root $\hat{\beta}_{2}=A-c_{K} / h+O\left(h^{-2}\right)$.

Proposition 3. Let $C_{0}$ be a space of continuous function with finite support. Define $K$ as the rectangle or Epanechnikov kernel. Then the operator $K_{h}$ is a contracting operator on the space $L_{2} \bigcap C_{0}$ with the $L_{2}$-norm.

To the proof of Proposition 3. The Fourier transformation for the rectangle kernel is $\tilde{K}(z)=(\sin z) / z$, and for the Epanechnikov kernel is $\tilde{K}(z)=$ $3(\sin z-z \cos z) / z^{3}$ with unique maximum value 1 at $z=0$. Thus, for the $n$-th iterative convolution, $\left\|K^{n}\right\|_{2} \rightarrow 0$ as $n \rightarrow \infty$. This confirms the result.

\section{A.2 Asymptotic Properties of LLP}

The asymptotic properties of local polynomial estimates are studied in Fan \& Gijbels (1996) and Härdle, Klinke \& Müller (1999). Under some smoothness conditions with bandwidth $h_{1}=k_{0} / n^{1 / 5}$ for a constant $k_{0}>0$, the results applied to our case provide the following formulas on the asymptotic normality

$$
\begin{gathered}
n^{2 / 5}\left\{\hat{m}_{h_{1}}(z)-m(z)\right\} \stackrel{\mathcal{D}}{\rightarrow} N\left(\frac{k_{0}^{2}}{2} \mu_{2}(K) \Delta m^{\prime \prime}(z), \frac{\|K\|_{2}^{2} \Delta \sigma^{2}(z)}{k_{0} p_{0}(z)}\right) \\
n^{2 / 5}\left\{\hat{\sigma}_{h_{1}}^{2}(z)-\sigma^{2}(z)\right\} \stackrel{\mathcal{D}}{\rightarrow} N \quad\left(\begin{array}{l}
\frac{k_{0}^{2}}{2} \mu_{2}(K)\left(\Delta\left(\sigma^{2}(z)\right)^{\prime \prime}+2\left(\Delta m^{\prime}(z)\right)^{2}\right) \\
\left.\frac{2 \Delta^{2} \sigma^{4}(z)\|K\|_{2}^{2}}{k_{0} p_{0}(z)}\right) .
\end{array}\right.
\end{gathered}
$$

Here $\mu_{2}(K)$ is the second moment of the kernel $K$ and $\|K\|_{2}$ is its $L_{2}$ norm. Furthermore $p_{0}(z)$ denotes the stationary density of $Z$ as given in Section 2.4.

\section{A.3 The Bootstrap Procedure}

The confidence bands in Section 2.6 for the nonparametric estimator are constructed by the following bootstrap method :

1. Choose a bandwidth $g$, which is larger than the optimal $h_{1}$ in order to have oversmoothing. Estimate then nonparametrically $m($.$) and \sigma^{2}($.$) and$ obtain the residual estimated errors :

$$
\hat{\varepsilon}_{i}=\frac{Y_{i}-\Delta \hat{m}_{g}\left\{Z^{\Delta}\left(t_{i}\right)\right\}}{\sqrt{\Delta} \hat{\sigma}_{g}\left\{Z^{\Delta}\left(t_{i}\right)\right\}} .
$$

Since we make the assumption that the $\varepsilon_{i}$ has zero-mean, we subtract the sample mean of $\hat{\varepsilon}_{i}$.

2. Replicate $N$ times the series of the $\left(\hat{\varepsilon}_{i}\right)$ with wild bootstrap obtaining $\left(\varepsilon_{i}^{*, n}\right)$ for $n=1, \ldots, N$ and build $N$ new bootstrapped series $\left(Z^{*, n}\right)$ :

$$
\begin{gathered}
Z_{1}^{*, n}=Z^{\Delta}\left(t_{1}\right) \\
Z_{i+1}^{*, n}-Z_{i}^{*, n}=\Delta \hat{m}_{g}\left(Z_{i}^{*, n}\right)+\sqrt{\Delta} \hat{\sigma}_{g}\left(Z_{i}^{*, n}\right) \varepsilon_{i}^{*, n} .
\end{gathered}
$$

Estimate again $m(z)$ and $\sigma^{2}(z)$ for each of the $N$ bootstrapped series with bandwidth $h_{1}$. 
3. Build the statistics:

$$
T_{m}^{*}=\sup _{z} \frac{\left|\hat{m}_{h_{1}}^{*, n}(z)-\hat{m}_{h_{1}}(z)\right|}{\hat{\sigma}_{h_{1}}^{*, n}(z)}
$$

and

$$
T_{\sigma}^{*}=\sup _{z}\left|\left(\hat{\sigma}^{2}\right)_{h_{1}}^{*, n}(z)-\hat{\sigma}_{h_{1}}^{2}(z)\right|
$$

4. Form the $(1-\alpha)$ confidence bands $K B$

$$
K B(m(.))=\left[\hat{m}_{h_{1}}(z)-\hat{\sigma}_{h_{1}}(z) t_{m, 1-\alpha / 2}, \hat{m}_{h_{1}}(z)+\hat{\sigma}_{h_{1}}(z) t_{m, \alpha / 2}\right]
$$

and

$$
K B\left(\sigma^{2}(.)\right)=\left[\hat{\sigma}_{h_{1}}^{2}(z)-t_{\sigma, 1-\alpha / 2}, \hat{\sigma}_{h_{1}}^{2}(z)+t_{\sigma, \alpha / 2}\right]
$$

where $t_{m, \alpha}$ and $t_{\sigma, \alpha}$ denote the empirical $\alpha$-quantile of $T_{m}^{*}$ and $T_{\sigma}^{*}$, respectively.

\section{Acknowledgments}

The authors acknowledge support by the University of Technology, Sydney and the Deutsche Forschungsgemeinschaft via Sonderforschungsbereich 373 "Quantifikation and Simulation ökonomischer Prozesse" at Humboldt-Universität zu Berlin.

\section{References}

Ait-Sahalia, Y. (1996), 'Nonparametric pricing of interest rate derivative securities.', Econometrica 64(3), 527-560.

Bibby, B. M. \& Sørensen, M. (1995), 'Martingale estimation functions for discretely observed diffusion processes', Bernoulli 1(1/2), 17 - 40.

Cox, J., Ingersoll, J. \& Ross, S. (1985), 'A theory of term structure of interest rates', Econometrica 2(53), 385-407.

Duan, J.-C. (1997), 'Augmented $\operatorname{GARCH}(p, q)$ process and its diffusion limit.', J. Econom. 79(1), 97-127.

Engle, R. F. \& Bollerslev, T. (1986), 'Modelling the persistence of conditional variances', Econometric Rev. 5(1), 1-87. With comments a reply by the authors.

Fan, J. \& Gijbels, I. (1996), Local Polynomial Modelling and its Applications Theory and Methodologies, Chapman and Hall, New York.

Fleming, W. H. \& Sheu, S.-J. (1999), 'Optimal long term growth rate of expected utility of wealth.', Ann. Appl. Probab. 9(3), 871-903. 
Föllmer, H. \& Schweizer, M. (1993), 'A microeconomic approach to diffusion models for stock prices.', Math. Finance 3(1), 1-23.

Franke, J., Kreiss, J.-P., Mammen, E. \& Neumann, M. (1998), Properties of the nonparametric autoregressive bootstrap. Discussion paper, SFB 373, No. 54/98, Humboldt University Berlin.

Frey, R. (1997), 'Derivative asset analysis in models with level-dependent and stochastic volatility.', CWI Q. 10(1), 1-34.

Gallant, A. \& Tauchen, G. (1996), 'Which moments to match?', Econometric Theory 12(4).

Genon-Catalot, V., Jeantheau, T. \& Larédo, C. (2000), 'Stochastic volatility models as hidden markov models and statistical applications', Bernoulli 6(6).

Ghysels, E., Harvey, A. \& Renault, E. (1996), Statistical Methods in Finance, Vol. 14 of Handbook of Statistics, North-Holland, chapter Stochastic Volatitlity, pp. 119-191.

Hall, P. (1985), 'Resampling a coverage pattern', Stoch. Proc. Appl. 20, 231-246.

Hansen, L. P. \& Scheinkman, J. A. (1995), 'Back to the future: Generating moment implications for continuous-time Markov processes.', Econometrica 63(4), 767-804.

Hansen, L. P., Scheinkman, J. A. \& Touzi, N. (1998), 'Spectral methods for identifying scalar diffusions.', J. Econom. 86(1), 1-32.

Härdle, W. (1990), Applied Nonparametric Regression, number 19 in 'Econometric Society Monographs', Cambridge University Press.

Härdle, W., Klinke, S. \& Müller, M. (1999), XploRe -The Statistical Computing Environment, Springer Verlag, New York.

Härdle, W. \& Tsybakov, A. (1997), 'Local polynomial estimators of the volatility function in nonparametric autoregression', Journal of Econometrics 81, 223-242.

Jiang, G. \& Knight, J. (1997), 'A nonparametric approach to the estimation of duffusion processes, with an application to a short-term interest rate model', Econometric Theory 13(5).

Karatzas, I. \& Shreve, S. E. (1998), Methods of Mathematical Finance, Vol. 39 of Applications of Mathematics, Stochastic Modelling and Applied Probability, Springer Verlag New York.

Kessler, M. \& Soerensen, M. (1999), 'Estimating equations based on eigenfunctions for a discretely observed diffusion process.', Bernoulli 5(2), 299-314.

Kloeden, P. E. \& Platen, E. (1999), Numerical Solution of Stochastic Differential Equations, Vol. 23 of Applications of Mathematics, Springer Verlag Berlin Heidelberg. 
Nelson, D. B. (1990), 'ARCH models as diffusion approximations', J. Econ. 45(1/2), 7-38.

Neumann, M. \& Kreiss, J.-P. (1998), 'Regression-type inference in nonparametric autoregression', Ann. Statist. 26, 1570-1613.

Platen, E. (2000), Risk premia and financial modelling without measure transformation. University of Technology Sydney, School of Finance \& and Economics and Department of Mathematical Sciences.

Platen, E. \& Rebolledo, R. (1996), 'Principles for modelling financial markets.', J. Appl. Probab. 33(3), 601-613. 